\title{
Mechanochemical synthesis of adsorbents based on silicon oxycarbide composites
}

\author{
I.S. Grishin, N.N. Smirnov \\ Department of Technology of Inorganic Substances, Faculty of Inorganic Chemistry and Technology, \\ Ivanovo State University of Chemistry and Technology, Ivanovo, Russia
}

\begin{abstract}
In the work, an attempt was made to mechanochemically synthesize silicon oxycarbide composites from activated carbon and silica. Structure of the composites was studied using powder X-ray diffraction and IR spectroscopy. Formation of silicon oxycarbides was confirmed by presence of Si-O-C bond. Influence of the raw materials ratio on structural and chemical properties of resulting composites was revealed. With an increase of silica share in the initial mixture, a decrease in specific surface area and pore volume was noted, as well as an increase of the concentration of surface functional groups. Samples of the composites were tested in processes of sorption of methyl orange and fluoride ions. It was established that adsorption capacity for methyl orange decreased, while that for fluoride ions significantly increased comparing to activated carbon.
\end{abstract}

\section{Introduction}

Silicon oxycarbide composites are usually considered as materials based on silicon oxycarbide (SiOC), which formally can be described as products of carbon incorporation into the network structure of silicates. The structure of silicon oxycarbide is a mixed network of silicon atoms tetrahedrally bonded to oxygen and carbon atoms. However, this network structure is different from nanosized silicon carbide and silicon dioxide, because it has the entire spectrum of structural units, which correspond to the general formula $\mathrm{SiO}_{\mathrm{x}} \mathrm{C}_{4-}$ x. According to that formula, there could be $\mathrm{SiO}_{4}, \mathrm{SiO}_{3} \mathrm{C}, \mathrm{SiO}_{2} \mathrm{C}_{2}, \mathrm{SiO}_{3} \mathrm{C}$, and $\mathrm{SiC}_{4}$ [1].

Currently, silicon oxycarbide are prepared from organosilicon polymers as raw materials, polyorganosiloxanes in particular. Synthesis of SiOC is carried out in several stages [2]. The first stage is crosslinking of thermoplastic precursors at $100-400^{\circ} \mathrm{C}$. As a result, an insoluble frame of the network structure is formed. The second stage is the thermal conversion of the frame into glass-ceramics at $600-1000^{\circ} \mathrm{C}$. Decomposition and subsequent removal of organic groups in the form of hydrocarbons and hydrogen takes place at that stage. The final stage is phase separation by crystallization at temperatures above $1200^{\circ} \mathrm{C}$. Silicon oxycarbides obtained by pyrolysis are characterized by heterogeneity at nano-level [1]. In addition to the structural units mentioned above, there is also a phase of segregated $\mathrm{sp}^{2}$-hybridized carbon in silicon oxycarbide. This carbon is highly disordered and turbostratic. Thus, there are two types of carbon in SiOC structure: carbide carbon, which is a part of the $\mathrm{sp}^{3}$ hybridization network, and $\mathrm{sp}^{2}$-hybridized carbon 
[3]. At present, the exact microstructure of SiOC has not yet been established. There are only model representations in literature [4,5].

To obtain SiOC-based composites, organosilicon precursors are usually modified first using various compounds containing a modifying agent. For the introduction of metals, their acetates or alcoholates are most often used. Another way is to select compounds that already contain the required modifying agent as synthesis precursors [6].

The unique structure of silicon oxycarbide composites determines all their properties, for example, high strength, chemical, and thermal stability. SiOC-based composites are used to produce a large number of different types of materials, in particular, coatings, tubes, fibers, etc. [7]. The most interesting are porous structures. Such composites are well suited as carriers of $\mathrm{Ni}, \mathrm{Co}$, and $\mathrm{Pt}$ for instance. The high catalytic activity of $\mathrm{Pt} / \mathrm{SiOC}$ composite in the process of conversion of $\mathrm{CO}$ to $\mathrm{CO}_{2}$ was noted, which was achieved by an improved distribution of Pt particles [8]. At the same time, silicon oxycarbide composites that do not possess significant specific surface area may still act as adsorbents. For example, they were used for wastewater treatment. Unlike activated carbon, the process of desorption of hydrocarbons [9] and methylene blue [10] from porous silicon oxycarbide proceeds easier, as well as their further regeneration. It was noted that HF-treated SiOC glass-ceramics effectively removes model dyes such as methylene blue, rhodamine B, and crystalline violet. However, the removal of metal ions such as $\mathrm{Cr}^{3+}, \mathrm{Pb}^{2+}$, and $\mathrm{Cd}^{2+}$ is quite limited [11].

Despite all the advantages of silicon oxycarbide and composites based on them, the key disadvantage is the high cost of their production. Firstly, this is due to expensive raw materials, and, secondly, to pyrolysis at high temperatures as the only synthesis method. In this regard, one of the aims of this work was to search for another method for producing silicon oxycarbide, and mechanochemical synthesis could potentially be the one. It is known that mechanical impact on solids accelerates chemical reactions. This process is highly effective for producing substances with unique properties [12]. Mechanical treatment is carried out mainly in grinding equipment, which includes mills of various types of action. It is considered that mechanical activation has an impulse character. Stress field occurrence and its relaxation are the key processes. One of the mechanical impact features is locality. That is, the stress field does not arise in the entire volume of a solid particle, but only at a place of its contact with another particle or grinding bodies. Deformation and destruction of solids lead to the formation of various energy-rich intermediate states, in which decomposition is accompanied by a significant heat release [13].

Another objective of the work was to study the adsorption activity of silicon oxycarbide composites obtained mechanochemically. In this case, adsorption activity could be a criterion of mechanochemical synthesis effectiveness for the production of adsorbents based on silicon oxycarbide composites.

\section{Experiments}

Activated carbon (AC) and silica (S) were chosen as raw materials for the mechanochemical synthesis of silicon oxycarbide composites. These materials are cheaper than organosilicon polymers. Activated carbon itself has a high specific surface area and is one of the most widely used adsorbents. Silica acts as a source of silicon. In addition, silanol groups that are present in silica could be potential centers for silicon oxycarbide formation under the intense mechanical impact.

The mechanochemical synthesis was carried out in a roller ring vibratory mill VM-4 with an energy intensity of $878 \mathrm{~W} / \mathrm{kg}$. Several mixtures of $\mathrm{AC}$ and $\mathrm{S}$ with various mass ratios of the components were subjected to mechanical treatment for $15 \mathrm{~min}$ (Table 1). 
Table 1. Samples information.

\begin{tabular}{lccccc}
\hline Sample & 1 & 2 & 3 & 4 & 5 \\
\hline AC:S mass ratio & $1: 0.2$ & $1: 0.5$ & $1: 0.7$ & $1: 1.2$ & $1: 2.0$ \\
Concentration of S, \% & 16.7 & 33.3 & 41.2 & 54.5 & 66.7 \\
\hline
\end{tabular}

The samples were analyzed using various experimental methods. The structure was investigated via powder X-ray diffraction. The analysis was performed using a DRON-3M diffractometer with the wavelength of $1.54059 \mathrm{~nm}\left(\mathrm{Cu}-\mathrm{K}_{\alpha}\right)$.

Both the raw materials and the samples obtained have a large number of different types of functional groups, which were characterized via IR Fourier spectroscopy. Spectra were taken on Bruker Tensor 27 FT-IR spectrometer by preparing tablets with $\mathrm{KBr}$. The measuring range was $400-4000 \mathrm{~cm}^{-1}$.

Porous structure was investigated using low-temperature nitrogen adsorption on SorbiMS device. Specific surface area and pore volume were calculated via the BET method.

The concentration of various surface functional groups was determined potentiometrically by the method described by [14].

Adsorption activity was determined using fluoride ions and methyl orange as compounds to be adsorbed. The adsorption capacity for fluoride ions was measured potentiometrically using a fluoride-selective electrode [15], and that for methyl orange was measured photometrically according to the method described by [16].

\section{$3 \quad$ Results and discussion}

The process of silicon oxycarbide synthesis is quite complicated because many chemical reactions proceed. The mechanism of SiOC formation and resulting product composition differ depending on the raw materials and synthesis method used. So, pyrolysis of organosilicon polymers is carried out in an inert medium. In this case, the product contains much more silicon and oxygen than carbon. Mechanochemical synthesis in the vast majority of cases implies air medium, in which oxygen is presented. The presence of the oxidizing agent doesn't allow silicon and carbon to bind directly to form silicon carbide that could occur during pyrolysis in an inert medium. Therefore, silicon and carbon are most likely to be bonded only through an oxygen bridge. This is possible due to the presence of various functional groups, in particular phenols, carboxyls, silanols, etc. Since activated carbon and silica were chosen as raw materials, it is possible to achieve the different compositions of silicon oxycarbides produced by varying AC:S mass ratio.

The formation of new compounds usually suggests structural changes. Figure 1 shows XRD patterns of activated carbon, silica, and also one of the products of their mechanochemical interaction with each other. Only one sample is given as an example since there are no visual changes in XRD patterns. It is seen that new phases do not occur. Both the raw materials and composites obtained are highly amorphous. XRD pattern of activated carbon shows reflections at 25 and $44^{\circ}$, which correspond to planes (002) and (101) in graphite [17]. A significant broadening of these reflections indicates a high degree of disorder in the structure of AC. Basal planes order of graphite is violated with microstrains and various defects. XRD pattern of silica is characterized by a large peak at $20^{\circ}$, which is due to amorphous silicon dioxide, as noted by [18]. 


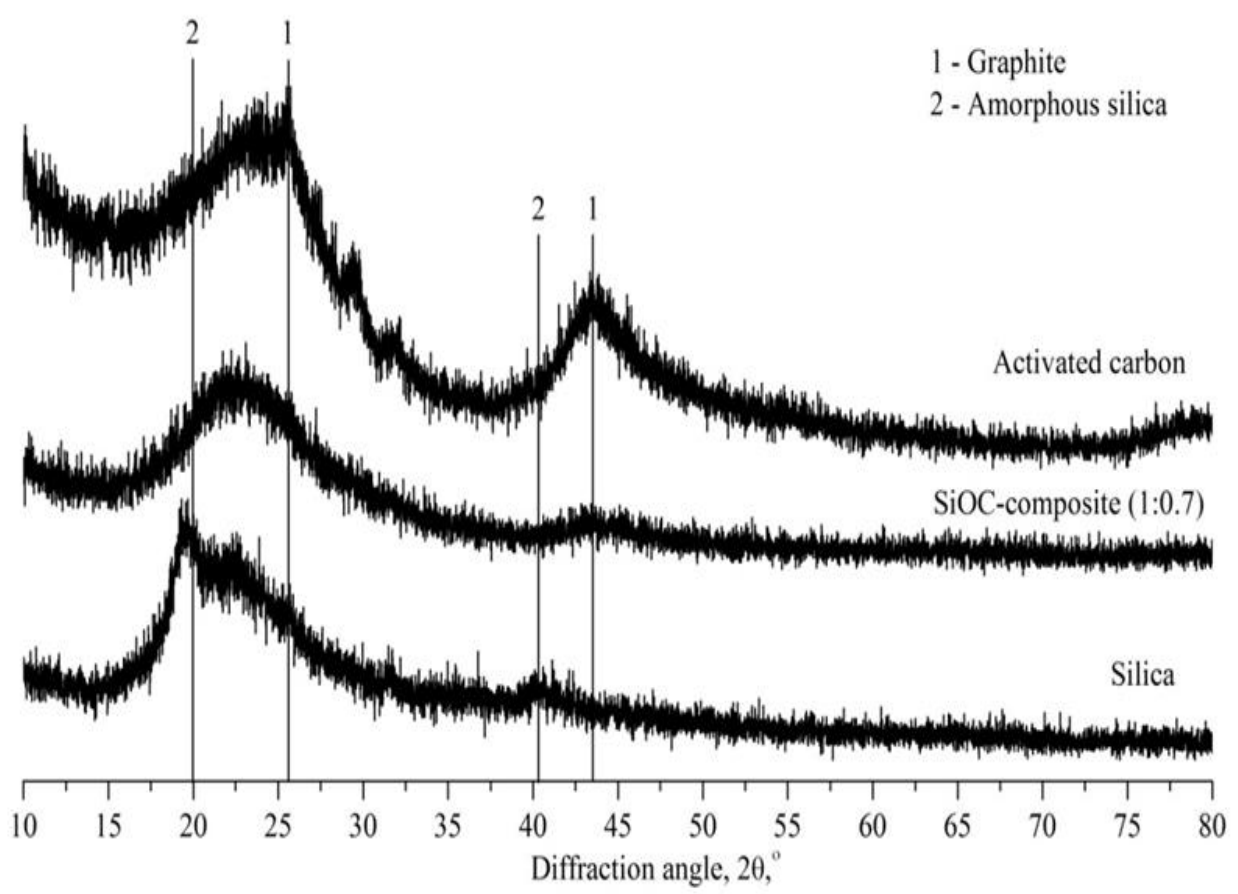

Fig. 1. XRD patterns of raw materials and one of the samples of SiOC composites.

It should be noted that profiles of the largest reflexes of activated carbon and silica are asymmetric. This may be the result of an overlay of reflexes with a bit different values of angle, width, and intensity, which correspond to different interplanar distances. Such an effect can be observed, in particular, due to zoning, structural heterogeneity of crystals, or to the presence of several metastable phases with different lattice parameters. At the same time, the reflex with the highest intensity on the XRD pattern of the SiOC composite has a lower degree of asymmetry. Obviously, this is due to the imposition of reflexes of AC and $\mathrm{S}$. It is noteworthy that a similar type of XRD pattern was obtained by other authors $[5,19]$ who used pyrolysis of organosilicon polymers for silicon oxycarbides synthesis.

Figure 2 shows the IR spectra of the samples with different AC:S mass ratios. There is a band in the region of $1630 \mathrm{~cm}^{-1}$ corresponding to vibrations of $\mathrm{C}=\mathrm{C}$ bonds in the aromatic system and $\mathrm{C}=\mathrm{O}$, for example, in carboxyl groups. This band could be due to adsorbed water as well. Vibrations in unsaturated aliphatic alkane groups give a $2850-2950 \mathrm{~cm}^{-1}$ band. Further up to $3200 \mathrm{~cm}^{-1}$ there is a wideband characterizing vibrations of the C-O bond. Finally, bands from 3400 up to $3700 \mathrm{~cm}^{-1}$ can be assigned to vibrations of phenol and silanol groups. It could also be vibrations in $\mathrm{H}_{2} \mathrm{O}$ molecules.

All of the bonds and groups mentioned above are obviously due to the presence of activated carbon. Characteristic bands for silica and silicon oxycarbide are located in the $400-1300 \mathrm{~cm}^{-1}$ region (Fig. 3). Basically, all bands, which are found for silicon oxycarbide obtained by pyrolysis, are concentrated in this region [5].

A cumulative band within $1000-1300 \mathrm{~cm}^{-1}$, which contains several overlaid bands, could serve as a confirmation of silicon oxycarbide formation. One of the bands lies around $1220 \mathrm{~cm}^{-1}$ and characterizes vibrations of Si-O-C bond. The other three bands in this region correspond to vibrations of Si-O bond in the cell, network, and linear types of structure. In fact, these are three-, two-, and one-dimensional structures that are present in amorphous silicon dioxide [20]. One can observe changes in the shape of this wide band, which are 
presumably caused by different structures of the composites, which in turn is a consequence of different $\mathrm{AC}: \mathrm{S}$ mass ratios.

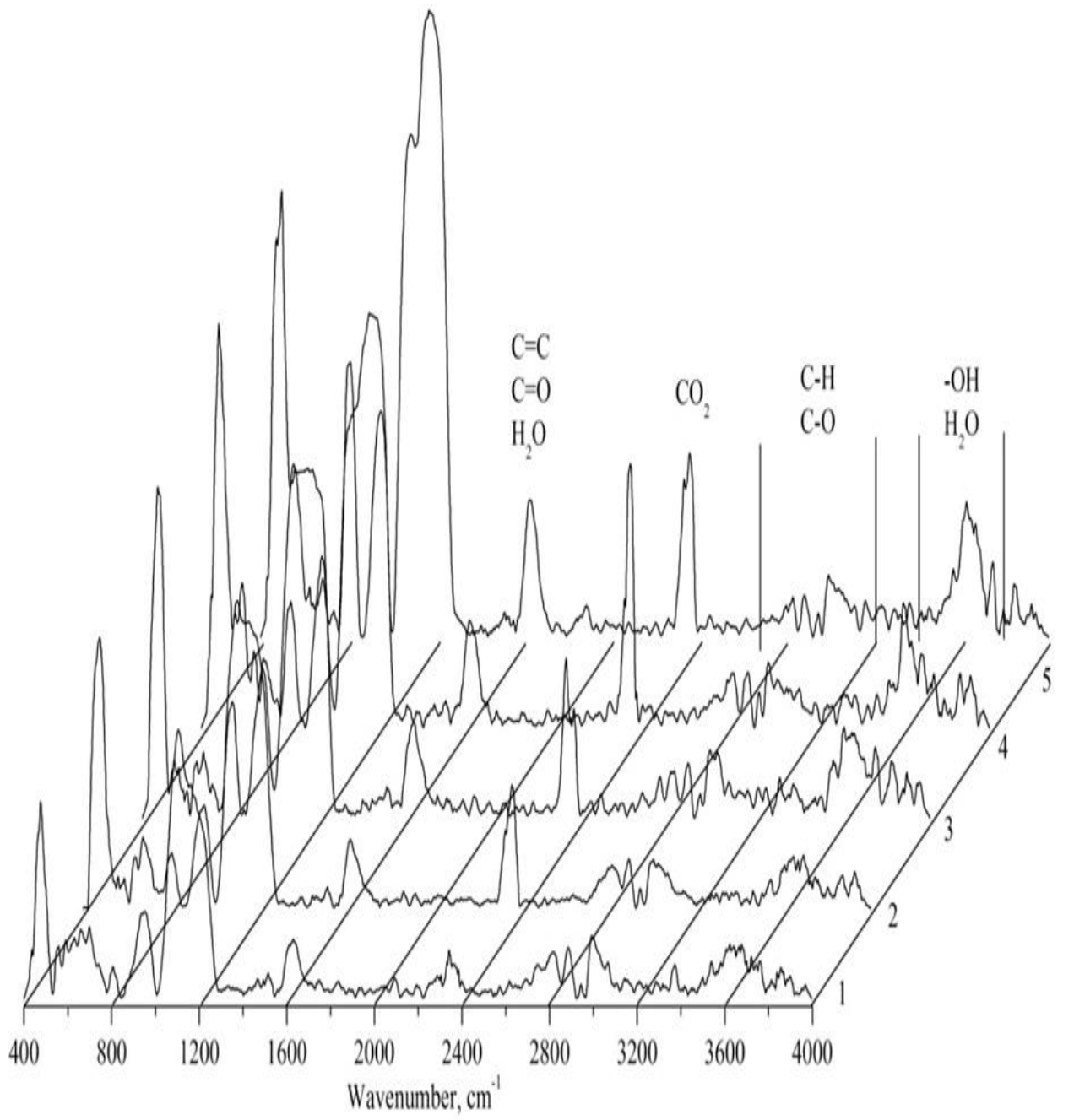

Fig. 2. IR spectra of samples of SiOC composites. 


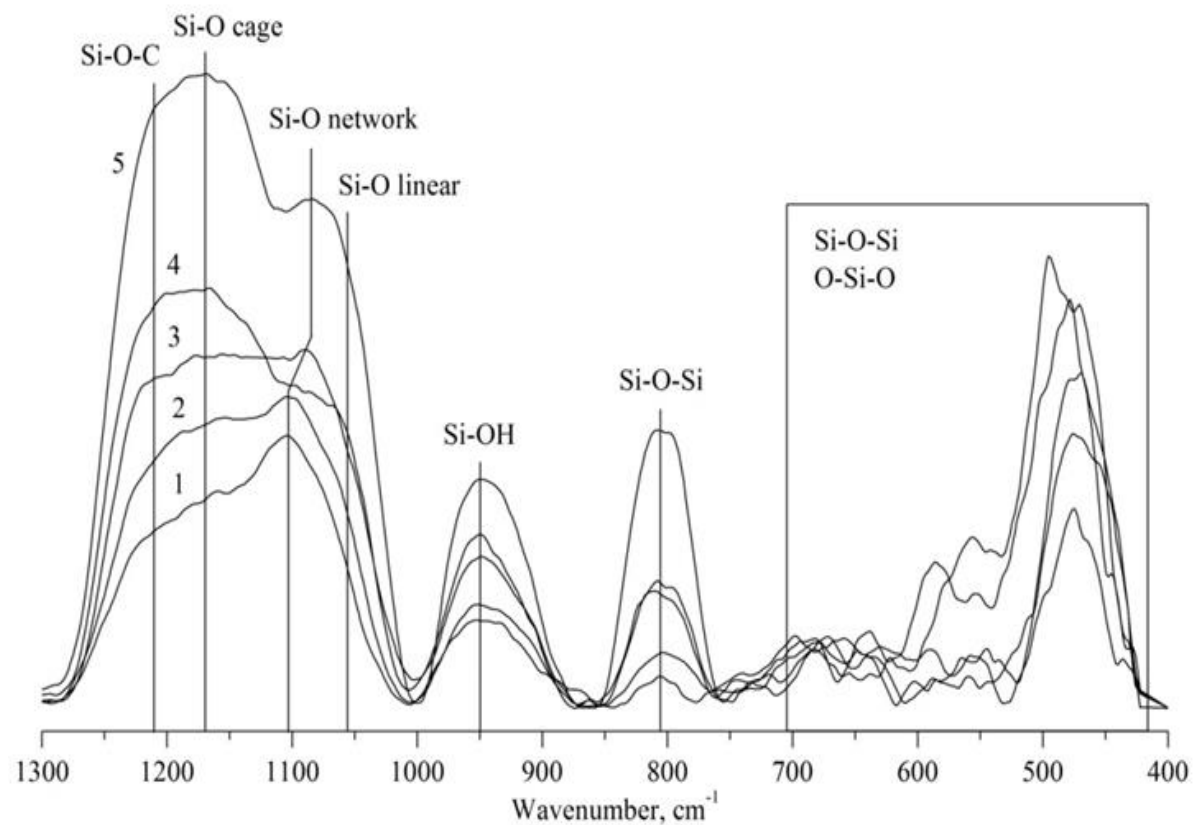

Fig. 3. IR spectra of samples of SiOC composites within $400-1300 \mathrm{~cm}^{-1}$ range.

When the concentration of silica is small, the prevailing band on the spectra is responsible precisely for network structure. Silicon oxycarbides are known to have network architecture [1]. Furthermore, with an increase of silica concentration in the initial mixtures, the intensity of the Si-O cage band increases significantly. This could mean layering of silicon dioxide particles on each other, as well as on activated carbon ones, which leads to the formation of three-dimensional structures. The band at $950 \mathrm{~cm}^{-1}$ is most often referred to as vibrations of silanols [21]. In the region of $800-820 \mathrm{~cm}^{-1}$, there is a band, which is identified differently in the literature. Some sources [5] characterize this band as vibrations of the $\mathrm{Si}-\mathrm{C}$ bond. However, as already noted, direct bonding of silicon and carbon is unlikely, when the oxidizing agent is present, although this is not impossible in principle [22] suggest that the band at $800 \mathrm{~cm}^{-1}$ corresponds to vibrations of Si-O-Si. Both bands may be present in the spectra forming a cumulative peak. Finally, bands in the region of $420-720 \mathrm{~cm}^{-1}$ in the literature [18] refer to vibrations of Si-O-Si and O-Si-O bonds.

The chemical structure also changes in the process of mechanochemical synthesis. This is primarily due to the oxidation of activated carbon with the formation of various surface functional groups. Table 2 presents the results of potentiometric titration, namely the concentration of various functional groups corresponding to a certain range of $\mathrm{pK}$.

Table 2. Results of potentiometric titration of SiOC composites.

\begin{tabular}{lllllllllll}
\hline & \multicolumn{3}{r}{2} & \multicolumn{3}{r}{3} & & \multicolumn{3}{c}{5} \\
\hline $\mathrm{pK}$ & $\mathrm{C}^{*}$ & $\mathrm{pK}$ & $\mathrm{C}$ & $\mathrm{pK}$ & $\mathrm{C}$ & $\mathrm{pK}$ & $\mathrm{C}$ & $\mathrm{pK}$ & $\mathrm{C}$ \\
$2.3-3.8$ & 0.188 & $2.2-4.9$ & 0.170 & $3.7-5.3$ & 0.096 & $3.3-4.7$ & 0.101 & $3.7-5.4$ & 0.097 \\
$4.3-5.4$ & 0.070 & $4.9-7.0$ & 0.139 & $5.3-7.1$ & 0.129 & $4.7-5.8$ & 0.060 & $5.4-7.3$ & 0.134 \\
$5.5-6.8$ & 0.131 & $7.0-8.5$ & 0.094 & $7.1-8.4$ & 0.088 & $5.8-7.3$ & 0.085 & $7.3-8.5$ & 0.070 \\
$6.9-8.0$ & 0.084 & $8.7-11.0$ & 0.456 & $8.4-11.2$ & 0.550 & $7.3-8.5$ & 0.065 & $8.5-9.9$ & 0.225 \\
$8.1-9.1$ & 0.060 & & & & & $8.5-9.9$ & 0.224 & $9.9-11.1$ & 0.467 \\
$9.5-10.2$ & 0.291 & & & & & $9.9-11.0$ & 0.461 & & \\
\hline
\end{tabular}


*Concentration expressed in $\mathrm{mmol} / \mathrm{g}$

In this case, several types of groups are possible: carboxyl (pK 3.8-5), phenol (pK approximately 10), and various silanols as well. The acidity of silanol groups depends on their structure [23]. So, there are isolated silanols (Si-OH) with pK 10.3-10.6, geminal silanols, which in turn can be convex and concave. That is, in this type of silanols, hydroxyls are located at different angles with respect to each other. Convex geminal silanols $\left(\mathrm{Si}=(\mathrm{OH})_{2}\right)$ are quite acidic, their $\mathrm{pK}$ is in the region of 2.3-3.5, concave geminal silanol ( $\mathrm{HO}-\mathrm{Si}-\mathrm{OH})$ are similar in acidity to isolated ones, and their $\mathrm{pK}$ is $8.5-9.5$. All the indicated types of silanols are observed on the pK spectrum of S (Fig. 4).

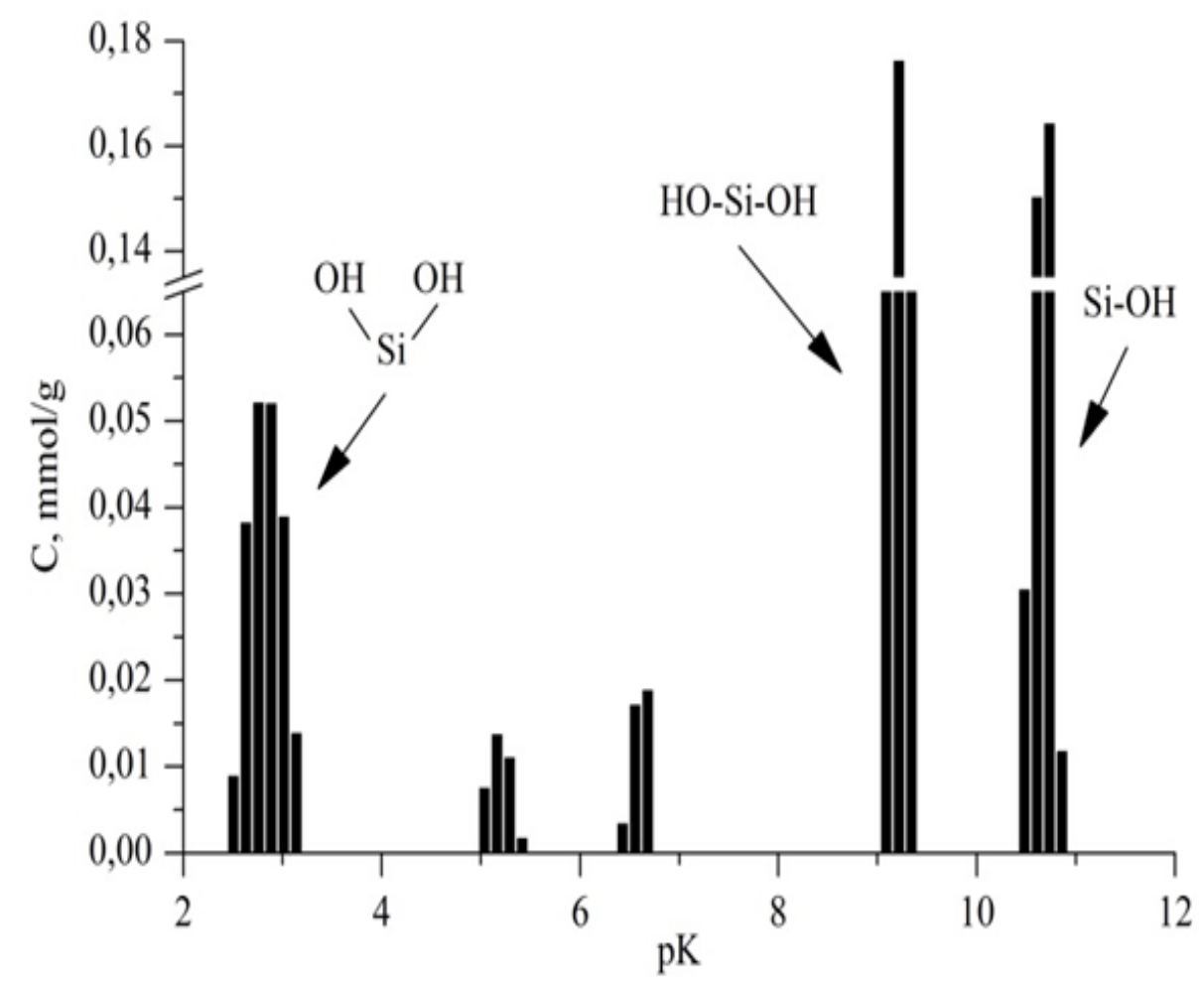

4. Functional groups of silica in the pK range of 2-12.

Fig.

Sample 1 contains mainly $\mathrm{Si}=(\mathrm{OH})_{2}$, as well as a small amount of HO-Si-OH. Sample 2 is the only one possessing all the indicated types of silanols. Convex geminal silanols are absent in samples 3-5. However, these samples have slightly acidic $\mathrm{Si}-\mathrm{OH}$ and $\mathrm{HO}-\mathrm{Si}-\mathrm{OH}$, and their concentration increases with an increase of silica share in the initial mixtures. In addition, the concentration of phenolic and carboxyl groups decreases, since activated carbon share decreases from sample 1 to sample 5.

Adsorption properties are primarily associated with the specific surface area. Table 3 shows the specific surface area $\left(\mathrm{S}_{\mathrm{sp}}\right)$ and pore volume $\left(\mathrm{V}_{\text {pore }}\right)$ of the samples and initial $\mathrm{AC}$. It can be seen that these parameters decrease as there is more silica in the initial mixtures. This was expected, since $A C$ is the one, which mostly contributes to $S_{\text {sp }}$ and $V_{\text {pore }}$, and its share decreases from sample 1 to sample 5. A similar trend is observed for pore volume. Nevertheless, even sample 5 having an AC fraction of about 33\% possesses a rather high specific surface area. Possibly, this is due to the formation of silicon oxycarbides and Si-O- 
Si network, which protects AC from significant destruction of its porous structure under the intensive mechanical impact.

Table 3. Specific surface area and pore volume of the samples.

\begin{tabular}{lcccccc}
\hline Sample & AC & 1 & 2 & 3 & 4 & \multicolumn{1}{c}{5} \\
\hline $\mathrm{S}_{\mathrm{sp}}, \mathrm{m}^{2} / \mathrm{g}$ & 1120 & 1051 & 983 & 902 & 834 & 798 \\
$\mathrm{~V}_{\text {pore }}, \mathrm{cm}^{3} / \mathrm{g}$ & 0.519 & 0.543 & 0.525 & 0.511 & 0.486 & 0.472 \\
\hline
\end{tabular}

Methyl orange and fluoride ions were chosen to test the synthesized composite materials as adsorbents. It is known that activated carbon is a good adsorbent possessing a developed porous structure. Its application implies physical adsorption in most cases. AC in general has a quite high adsorption capacity for methyl orange [24]. At the same time, AC isn't very effective for fluoride ions removal, and silicon-containing materials are often used for this process, silica in particular. This is because removal of fluoride ions considers ion exchange [25]. For this to happen $\mathrm{S}$ possesses a significant number of functional groups on its surface that was mentioned above. The composites were tested with adsorption of methyl orange and fluoride ions to show their effectiveness in both processes.

Testing procedures of the synthesized composite materials showed that the adsorption capacity of the samples for methyl orange decreased from sample 1 to sample 5 (Fig. 5). This is mainly due to a decrease of specific surface area since methyl orange is adsorbed physically. At the same time, the adsorption capacity for fluoride ions increased significantly. This tendency could be explained by an increase of concentration of surface functional groups, primarily containing - $\mathrm{OH}$ fragments, which is capable of exchange with fluoride ions. These are phenolic groups, as well as hydroxyls in $-\mathrm{COOH}$, and silanols.

Adsorption capacities of initial AC for methyl orange and fluoride ions are 176.4 and 9.6 $\mathrm{mg} / \mathrm{g}$, respectively. Comparing AC and sample 5, it can be noted that adsorption capacity for methyl orange decreased by $65.3 \%$, and for fluoride ions increased by $131.1 \%$.

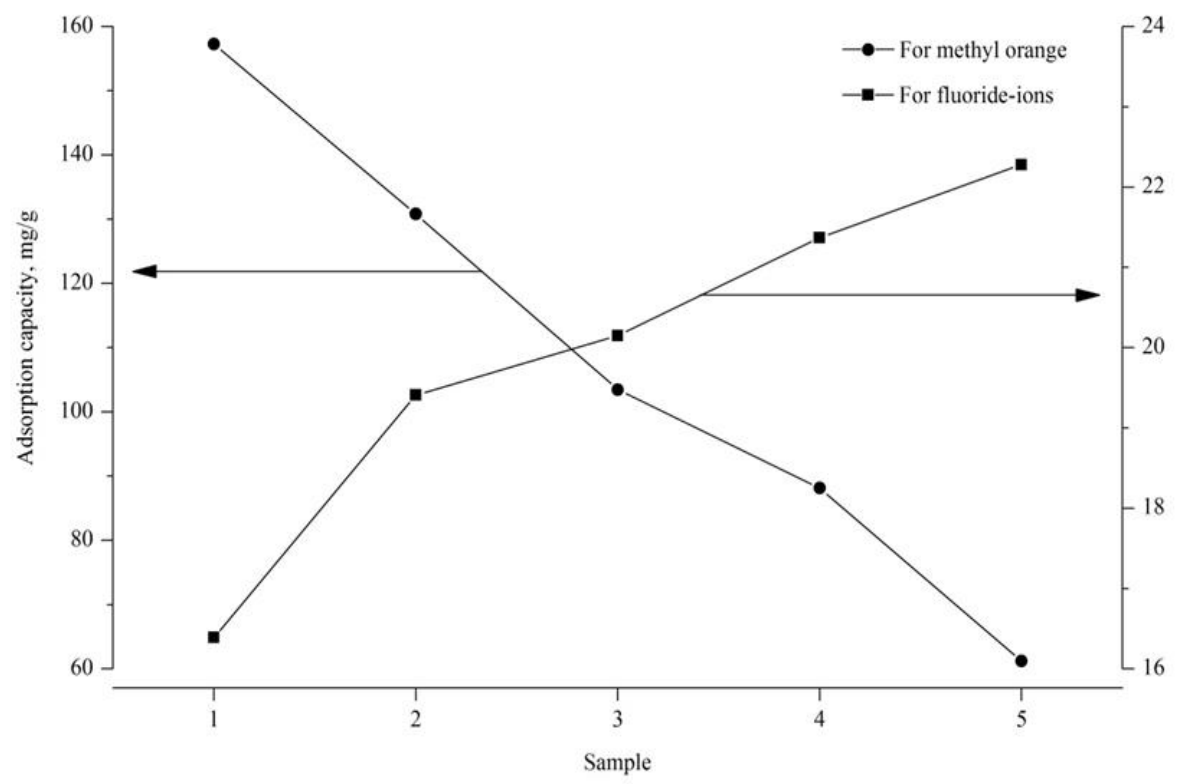

Fig. 5. Adsorption capacity of the samples for methyl orange and fluoride ions. 


\section{Conclusions}

Mechanochemical synthesis is effective because the mechanical impact could make chemical reactions, which aren't possible at standard conditions, occur. A process like this is the production of silicon oxycarbide that requires high temperatures, as well as expensive organosilicon polymers as raw materials. In this work, it was shown that an intensive supply of mechanical energy allows one to obtain silicon oxycarbide even from activated carbon and silica. However, in this case, silicon oxycarbide are an intermediate phase between $\mathrm{AC}$ and $\mathrm{S}$. Therefore, it is possible to mechanochemically produce exactly silicon oxycarbide composites, which, upon further processing, could be converted directly to silicon oxycarbide themselves. This may be useful if a specific composition of silicon oxycarbide is required.

Mechanochemical synthesis of SiOC composites proceeds together with the oxidation of activated carbon, which increases the concentration of various functional groups, in particular phenolic and carboxylic ones. Probably, the formation of Si-O-C bonds occurs due to these groups. The structure of the composites obtained varies depending on the initial mixture composition. In fact, the larger the proportion of silica, the more Si-O-C bonds could form. This trend is observed in absolute terms. However, the degree of binding of $\mathrm{AC}$ and $\mathrm{S}$ decreases, since silica excess overlaps the network structure of silicon oxycarbide by crosslinking of $\mathrm{Si}-\mathrm{OH}$ bonds to form $\mathrm{Si}-\mathrm{O}-\mathrm{Si}$. From this point of view, a small concentration of $\mathrm{S}$ in the initial mixture is required to achieve a high degree of binding of $\mathrm{AC}$ and $\mathrm{S}$.

Assessing the adsorption properties of the obtained composites, it should be noted that the role of silicon oxycarbide in sorption processes is not fully revealed. A decrease in specific surface area was observed with the addition of S to AC. Nevertheless, the specific surface area of even pure AC under mechanical impact would decrease due to the destruction of its porous structure. This decrease of the specific surface area is responsible for the reduction of adsorption capacity for methyl orange. At the same time, it was observed that the adsorption capacity for fluoride ions significantly increased. It can be assumed that synthesized silicon oxycarbide composites can act as ion exchangers. However, further studies are needed to confirm this and to investigate the process deeper.

This work was supported by the Ministry of Science and Higher Education of the Russian Federation (Project № FZZW-2020-0010). Equipment of the Center of Collective Use of Ivanovo State University of Chemistry and Technology was used when carrying out the research.

\section{References}

1. C. Stabler, E. Ionescu, M. Graczyk-Zajac, I. Gonzalo-Juan, R. Riedel Silicon oxycarbide glasses and glass-ceramics: "All-Rounder" materials for advanced structural and functional applications. Journal of the American Ceramic Society, 101, 4817-4856 (2018)

2. Belot, V., Corriu, R.J.P., Leclercq, D., Mutin, P.H. \& Vioux, A. Thermal redistribution reactions in crosslinked polysiloxanes. Journal of Polymer Science Part A: Polymer Chemistry, 30(4), 613-623 (1992)

3. F. Roth, C. Schmerbauch, E. Ionescu, N. Nicoloso, O. Guillon, R. Riedel High-temperature piezoresistive $\mathrm{C} / \mathrm{SiOC}$ sensors. Journal of Sensors and Sensor Systems, 4, 133-136

4. S. Sen, S. Widgeon On the mass fractal character of Si-based structural networks in amorphous polymer-derived ceramics. Nanomaterials, 5(1), 366-375 (2015) 
5. E. Ionescu, C. Linck, C. Fasel, M. Muller, H. Kleebe, R. Riedel Polymer-derived $\mathrm{SiOC} / \mathrm{ZrO}_{2}$ ceramic nanocomposites with excellent high-temperature stability. Journal of the American Ceramic Society, 93(1), 241-250 (2010)

6. P. Colombo Engineering porosity in polymer-derived ceramics. Journal of the European Ceramic Society, 28(7), 1389-1395 (2008)

7. M. Adam, C. Vakifahmetoglu, P. Colombo, M. Wilhelm, G. Grathwohl Ceramics Functional materials for adsorption techniques. Journal of the European Ceramic Society, 25, 271-276 (2013)

8. J. Pan, J. Ren, Y. Xie, X. Wei, Y. Guan, X. Yan, H. Tang, X. Cheng Porous SiOC composites fabricated from preceramic polymers and wood powders for efficient dye adsorption and removal. Research on Chemical Intermediates, 43, 3813-3832 (2017)

9. D. Zeydanli, S. Akman, C. Vakifahmetoglu Polymer-derived ceramic adsorbent for pollutant removal from water. Journal of the American Ceramic Society, 101(6), 22582265 (2018)

10. Takacs, L. 2013. The historical development of mechanochemistry. Chemical Society Reviews, 42(18), 7649-7659.

11. V. Sepelak, A. Duvel, M. Wilkening, K.-D. Becker, P. Heitjans Mechanochemical reactions and syntheses of oxides. Chemical Society Reviews, 42(18), 7507-7520 (2013)

12. I. I. Salame, T. J. Bandosz Surface chemistry of activated carbons: combining the results of temperature-programmed desorption. Boehm. and potentiometric titrations. Journal of Colloid and Interface Science, 240(1), 252-258 (2001)

13. T. Akafu, A. Chimdi, K. Gomoro Removal of fluoride from drinking water by sorption using diatomite modified with aluminum hydroxide. Journal of Analytical Methods in Chemistry, 1-11 (2019)

14. S. Rattanapan, J. Srikram, P. Kongsune Adsorption of methyl orange on coffee grounds activated carbon. Energy Procedia, 138, 949-954 (2017)

15. Tzeng, S.S., Hung, K.H. \& Ko, T.H. Growth of carbon nanofibers on activated carbon fiber fabrics. Carbon, 44(5), 859-865 (2006)

16. M. Nabil, K. R. Mahmoud, A. El-Shaer, H.A. Nayber Preparation of crystalline silica (quartz, cristobalite, and tridymite) and amorphous silica powder (one step). Journal of Physics and Chemistry of Solids, 121, 22-26 (2018)

17. J. Feng, Y. Xiao, Y. Jiang, J. Feng Synthesis, structure, and properties of silicon oxycarbide aerogels derived from tetraethylorthosilicate/polydimethylsiloxane. Ceramics International, 41, 5281-5286 (2015)

18. M. A. Mazo, A. Tamayo, J. Rubio Advanced silicon oxycarbide-carbon composites for high-temperature resistant friction systems. Journal of the European Ceramic Society, 36(10), 2443-2452 (2016)

19. S. Yasuhara, T. Sasaki, T. Shimayama, K. Tajima, H. Yano, S. Kadomura, M. Yoshimaru, N. Matsunaga, S. Samukawa Super-low-k SiOCH film $(k=1.9)$ with extremely high water resistance and thermal stability formed by neutral-beamenhanced CVD. Journal of Physics D: Applied Physics, 43(6), 65203-65210 (2010)

20. J. Liao, P. Gao, L. Xu, J. Feng A study of morphological properties of $\mathrm{SiO}_{2}$ aerogels obtained at different temperatures. Journal of Advanced Ceramics, 7(4), 307-316 (2018) 
21. G. F. Andrade, D. C. F. Soares, R. K. de S. Almeida, E. M. B. Sousa Mesoporous silica SBA-16 functionalized with alkoxysilane groups: preparation, characterization, and release profile study. Journal of Nanomaterials, 1-10 (2012)

22. X. Liu, J. Cheng, X. Lu, R. Wang Surface acidity of quartz: understanding the crystallographic control. Physical Chemistry Chemical Physics, 16(48), 26909-26916 (2014)

23. G. Crini Non-conventional low-cost adsorbents for dye removal: A review. Bioresource Technology, 97(9), 1061-1085 (2006)

24. M. Habuda-Stanić, M. E. Ravančić, A. Flanagan A Review on Adsorption of Fluoride from Aqueous Solution. Materials (Basel, Switzerland), 7(9), 6317-6366 (2014) 